\title{
Focus on neuroscience methods
}

\author{
In this special issue, we present a series of reviews, perspectives and commentaries that highlight advances in \\ methods and analytical approaches and provide guidelines and best practices in various areas of neuroscience.
}

$\mathrm{N}$ euroscience is continuously evolving, as technologies and analytical approaches-from the molecular and cellular levels to the systems and behavioral levels-open up previously inaccessible research questions and strategies. Understanding the transformative aspects of new approaches, as well as their limitations, is central to the progress that these techniques enable. Nature Neuroscience presents a special focus issue that highlights advances in methods, analyses and practices across scales of investigation and subfields of neuroscience.

Single-cell transcriptomics has enabled detailed profiling of cellular heterogeneity in the brain, but it is equally important to arrive at principled approaches to organize and interpret those data. Rafael Yuste, Michael Hawrylycz, Hongkui Zeng, Ed Lein and colleagues discuss the systematic classification of cortical cell types based on transcriptomics and the orthogonal modalities of morphology, physiology and connectivity, and they propose the implementation of a community platform for aggregating and updating the taxonomies in a standardized manner.

The analysis of cell types illustrates the complexity of brain organization. Three-dimensional tissue culture has propelled the modeling of human brain development in healthy and disease states. Ilaria Chiaradia and Madeline Lancaster provide an overview of brain organoids as a research tool and discuss their use in the study of brain development and disease. The authors further highlight technical innovations and advances in protocols used to generate brain organoids, as well as future avenues for modeling with organoids.

Human-derived induced pluripotent stem cells (iPSC) have led to the development of improved models of brain disorders, but functionally characterizing disease-associated genomic loci remains a challenge. Kayla Townsley, Laura Huckins and Kristen Brennand discuss approaches for assessing the functional effects of psychiatric and neurodevelopmental disease candidate risk variants, including how they may be localized to specific cell types and how they may affect gene expression.

The analysis of brain function also calls for ways to probe neural activity in vivo. Extending the scales of measurement and types of questions that can be addressed in behaving animals has necessitated the development of new technologies. John Rogers and colleagues review advances in electrical, optical and microfluidic devices and sensors for recording and manipulating neural signals. The authors highlight cutting-edge engineering and novel systems that can be implemented wirelessly in freely moving animals.

An invaluable window through which to view the brain is the analysis of behavior. Recent developments have transformed the ways in which behavioral measurement can be achieved. Talmo Pereira, Joshua Shaevitz and Mala Murthy provide an overview of techniques for automated quantification of behavior in animals, including deep-learningbased pose estimation methods and approaches for extracting and classifying behavioral dynamics. The authors discuss ways to relate quantitative behavioral analyses to recordings of neural activity, a strategy that can provide insights into neural coding.

The noninvasive imaging of spontaneous neural activity has yielded insights into functional brain network organization. Janine Bijsterbosch, Eugene Duff and colleagues discuss analytical approaches to represent functional connectivity data and, importantly, consider how the choice of representation can shape the interpretation of functional brain organization. The authors provide guidelines aimed at improving generalizability and reproducibility in the field.
Data acquisition, analysis and reporting can all influence research reproducibility and replicability. In their perspective, Cyril Pernet, Aina Puce and their colleagues from the COBIDAS MEEG committee discuss issues related to electroencephalography (EEG) and magnetoencephalography (MEG) studies, and they provide best practice recommendations to promote interpretability, sharing and reuse of these data.

We assembled this special issue to highlight techniques and analytical approaches that facilitate research across diverse areas of neuroscience, together with recommendations and guidelines for best practices. This issue also contains several Technical Reports that describe novel methods and approaches, including genetic tools for selective labeling and manipulation of cells, imaging for neuronal circuit reconstruction, and analysis of electrophysiological recordings and network functional connectivity. With this, we aim to signal our continued interest in publishing transformative technologies and methods in neuroscience and our commitment to enhancing data and analytical reporting and reproducibility. This collection represents only a small sample of the broad array of approaches that are enabling new discoveries in neuroscience. Exciting advances in genetic strategies for targeting cell populations, optical methods for recording and manipulating neuronal ensembles in behaving animals, and computational and analytical approaches for interpreting large-scale brain activity are among the many types of developments that we anticipate will facilitate new insights in the near future. We look forward to hearing about these and other novel advances as they continue to push neuroscience into interesting new directions.

Published online: 23 November 2020 https://doi.org/10.1038/s41593-020-00750-Z 